OPEN

SUBJECT AREAS:

CELL BIOLOGY

PLANT POLARITY

MICROFLUIDICS

Received

11 April 2014

Accepted

8 July 2014

Published

28 July 2014

Correspondence and requests for materials should be addressed to P.N. (peter.nick@kit.

edu)

\section{Plant Cells Use Auxin Efflux to Explore} Geometry

\author{
Beatrix Zaban' ${ }^{1}$ Wenwen Liu ${ }^{2}$, Xingyu Jiang ${ }^{2} \&$ Peter Nick'
}

${ }^{1}$ Molecular Cell Biology, Botanical Institute, Karlsruhe Institute of Technology, Kaiserstr. 2, D-76128 Karlsruhe, Germany, ${ }^{2}$ CAS Key Lab for Biological Effects of Nanomaterials and Nanosafety, National Center for NanoScience and Technology, 11 Beiyitiao, ZhongGuanCun, Beijing, China.

Cell movement is the central mechanism for animal morphogenesis. Plant cell development rather relies on flexible alignment of cell axis adjusting cellular differentiation to directional cues. As central input, vectorial fields of mechanical stress and gradients of the phytohormone auxin have been discussed. In tissue contexts, mechanical and chemical signals will always act in concert; experimentally it is difficult to dissect their individual roles. We have designed a novel approach, based on cells, where directionality has been eliminated by removal of the cell wall. We impose a new axis using a microfluidic set-up to generate auxin gradients. Rectangular microvessels are integrated orthogonally with the gradient. Cells in these microvessels align their new axis with microvessel geometry before touching the wall. Auxin efflux is necessary for this touch-independent geometry exploration and we suggest a model, where auxin gradients can be used to align cell axis in tissues with minimized mechanical tensions.

lant morphogenesis cannot rely on cell movement, because plant cells are encased by a cellulosic wall. Therefore, plants use a flexible alignment of their cell axis as major mechanism for morphogenetic changes. As they face a hypotonic environment, they expand by uptake of water and are pressed against the cell wall. This so called turgor pressure can accumulate to considerable tissue tensions, which on the one hand can be used as signal for integration of the body plan (for review see 1), but on the other have to be minimized in order to ensure tissue stability. A simple and elegant mechanism to release mechanical tension would be to orient cell axis with respect to force. In fact, the compression of plant cells in tissue culture aligns the new cell plates with the force vector ${ }^{2}$.

Phyllotaxis has been intensively studied as a model for morphogenetic responses through axis adjustment. In the growing meristem, new leaf primordia are laid down in a certain distance from preexisting leaves, leading to characteristic patterns that are under genetic control. This phenomenon has long been explained on a biophysical base: the older primordia would, due to increase tissue tension, suppress the formation of new primordia in their neighborhood. On the base of this mechanism, the position of incipient primordia could be predicted by the modeled stress-strain patterns (for a classical review see 3). This biophysical explanation was supported by work, where local release of tension using beads coated with extensin, a protein that softens the cell-wall, inverted the phyllotactic pattern ${ }^{4}$. Moreover, in-vivo imaging of GFP-tagged microtubules (as early readout of cell axis adjustment), in combination with ablation of meristem layers produced responses that were matched by stress-strain modeling ${ }^{5}$. However, there exist concurrent models, where the preexisting primordia drain their neighborhood from diffusible auxin such that no further primordia can be initiated ${ }^{6}$. This chemical model is supported by experiments, where apical meristems had been freed from any primordia by treatment with inhibitors of auxin transport, such that it was possible to generate and manipulate de-novo patterns of phyllotaxis by local application of either auxin or auxin-transport inhibitors ${ }^{7}$. The central player in the chemical model is the auxin-efflux regulator PIN1, although the observation of a residual pattern in the pin1 mutant suggests that additional factors participate in patterning ${ }^{8}$. A temporal separation between induction and manifestation of directionality has also been found for the Fucus zygote ${ }^{25}$. The Fucus system has been for long time the best understood model for polarity induction, but it illustrates the general feature of symmetry break, although the phaeophycean algae do not fall into the ancestral line of terrestrial plants and therefore probably have developed polarity induction by convergent evolution.

The phyllotaxis case shows exemplarily that mechanical and chemical signals probably act in concert on the adjustment of cell axis. This calls for systems that are simpler than a multilayered tissue, such that these signals can be investigated separately. This consideration motivated us to design a system, where generation of axis and polarity can be generated de novo in single cells. To return to such a tabula-rasa state, we eliminated axis and 

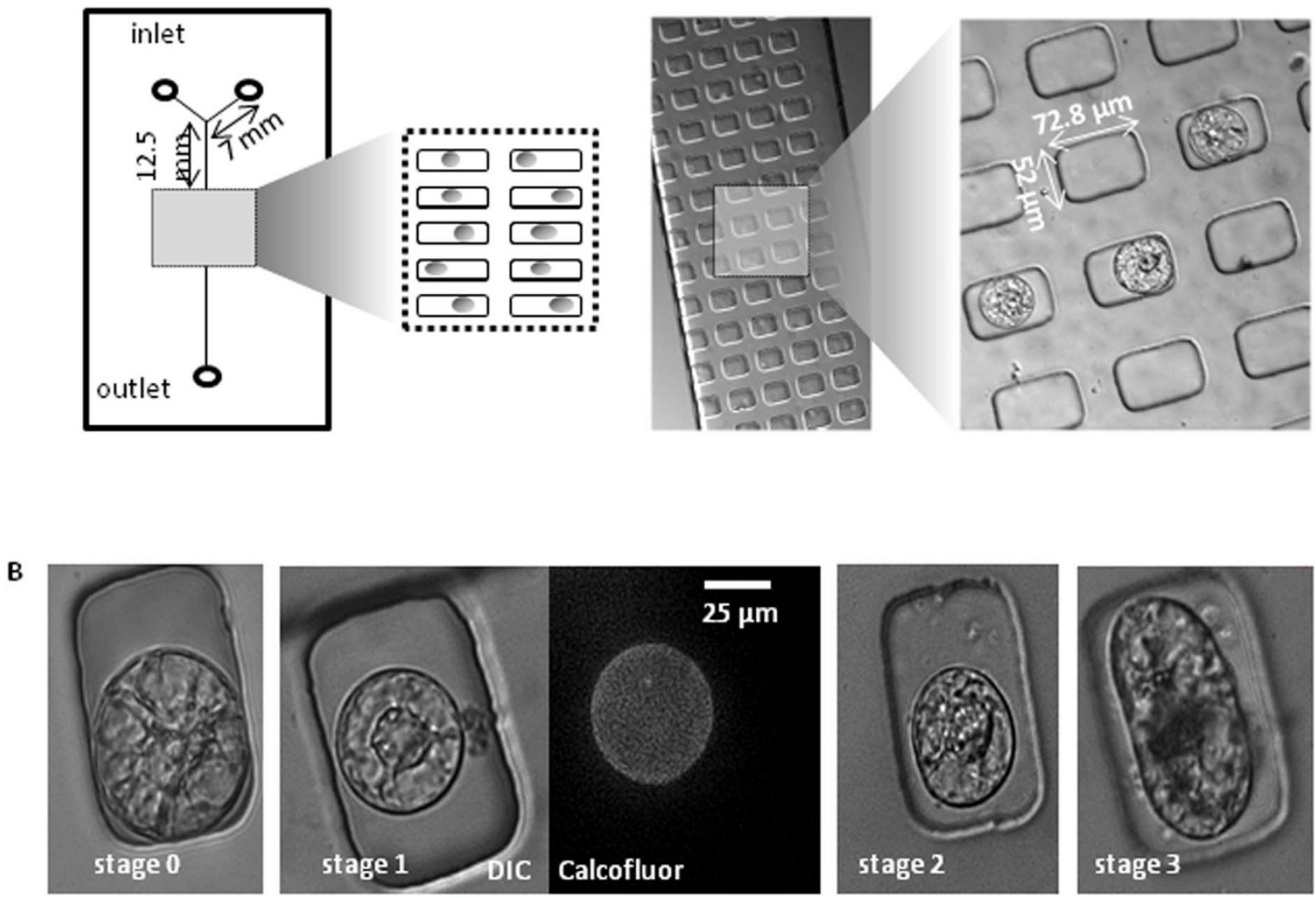

Figure $1 \mid$ Microfluidic chamber for regeneration of protoplasts in microvessels. (A) Design of channel and microvessels and representative images of microvessels prior to (left) and subsequent to (right) filling with protoplasts. (B) Representative images for different stages of protoplast regeneration following the classification given in (9). Stage 0 (days 0-1) protoplast with central nucleus, from which numerous fine cytoplasmic strands emanate, nuclear position is vividly changing during that stage (days $0-1$ ), stage 1 (around day 1) symmetrical cell with central nucleus apparently tethered to few, massive strands, a cellulosic wall is present at this stage and can be labelled by Calcofluor White, stage 2 (around day 2) first appearance of axiality manifest as ovoid shape, stage 3 (from day 3 ) rapid cell expansion parallel to this axis.

polarity by removal of the cell wall in tobacco BY-2 cells, and then triggered the formation of a new axis and polarity'. By fluorescently tagged transgenic marker lines we could follow the behavior and the role of the cytoskeleton during this phenomenon. Using anticytoskeletal compounds, as well as inducible expression of actinbundling proteins, we showed that a dynamic population of actin was necessary for cell polarity, whereas cell axis was dependent on microtubules.

In the current work, we integrate this tabula-rasa approach into a microfluidic platform ${ }^{10}$, which provides directional chemical cues by the flow of auxin-containing medium through the system and mechanical cues by the preformed geometries of the rectangular microvessels. Based on this approach, we observe that a new cell axis aligns with the geometry of the microvessel before the cell touches the wall, i.e. before there is a mechanical input. To get insight into the underlying mechanism, we interfered either by providing a perpendicular flux of auxin, or by inhibition of auxin efflux through the specific inhibitor 1-N-naphthyl-phthalamic acid (NPA). The results support a model, where plant cells use auxin efflux to explore the geometry of their environment and to align their axis at an early stage such that during subsequent expansion the mechanical tension in the tissues is minimized.

\section{Results}

A microfluidic system to study plant axis formation de novo. To impose chemical or mechanical cues to the regenerating protoplasts, a microfluidic system was designed. It consisted a Y-shaped channel allowing for influx of medium and rectangular microvessels inserted into the bottom of the channel such that the long axis of the microvessel was oriented perpendicular with the flow of medium (Fig. 1A). To accommodate the technical prerequisites- like the accessibility for microscopic inspection, the suppression of protoplast floating, the homogeneity of the microvessels, sterility, the exclusion of template autofluorescence and leakage- with the biological requirements (sufficient supply of nutrients, high viability), the fabrication strategy, material, size and depth of vessels as well as width and depth of the channel, furthermore the speed of flow and the viscosity of the medium had to be adjusted during lengthy preparatory experiments. This optimization procedure led to a system, where around $90 \%$ of loaded protoplasts were found viable even after 7 days of culture in the microvessels. These protoplast passed through the characteristic sequence of axis formation (Fig. 1B) starting from a spherical protoplast (stage 0), over a radial symmetric cell with a cell wall that can be fluorescently stained by Calcofluor (stage 1), followed by a break of radial symmetry and appearance of axiality (stage 2), and eventually, directional cell expansion (stage 3). Only $12 \%$ of the cells did not regenerate a wall and remained arrested in stage 0 , although maintaining viability. Although this fraction of non-regenerating cells is higher than the 5-7\% found for axis regeneration outside a microfluidics environment ${ }^{9}$, the fact that almost $90 \%$ of viable cells underwent normal axis formation shows that conditions have been tuned close to the technically possible optimum.

Cell axis aligns with vessel geometry before touching the wall. For the majority of regenerating protoplasts, the cell axis aligned with the 

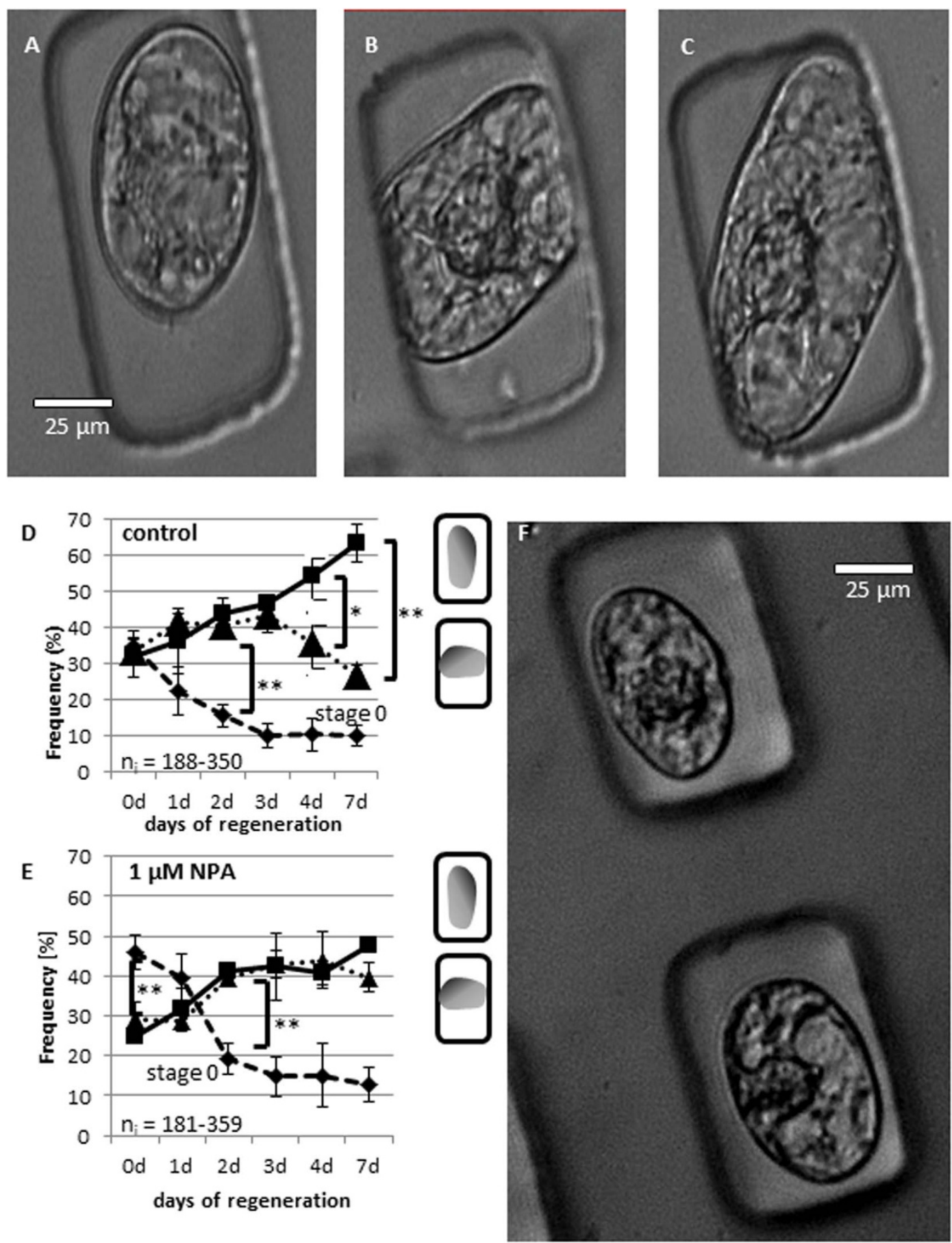

Figure 2 Cell axis aligns with vessel geometry before touching the wall. (A-C) different orientations of cell axis with respect to microvessel geometry: (A) longitudinal, (B) transverse, (C) cell during tilting from transverse to longitudinal orientation. (D), (E) Temporal change in the incidence of longitudinal (squares) and transverse (triangles) cells, as well as protoplasts that have not initiated regeneration (stage 0 , diamonds) under control conditions (D) or in presence of $1 \mu \mathrm{M}$ of the auxin efflux inhibitor 1-N-Napthylphthalamic acid (NPA, E). Data represent average values and standard errors from three independent biological replicas. Brackets represent statistical significance of the indicated differences as tested by a paired, two-sided ttest $\left(* P<5 \%,{ }^{*} P<1 \%\right.$ ). (F) Representative image for the longitudinal alignment of cell in stage 2 that is aligned prior to touching the wall of the microvessel.

longer axis of the rectangular micro-vessel, subsequently termed as "longitudinal orientation" (Fig. 2A). Only a smaller fraction produced an axis parallel with the shorter axis of the chamber, subsequently termed as "transverse orientation" (Fig. 2B). When these transversely oriented cells touched the wall of the microvessel, they progressively tilted over an oblique intermediate state (Fig. 2C) into a longitudinal orientation, such that the fraction of these cells further drops between day 4 , when most of the transversely oriented cells touched the wall, and day 7 (Fig. 2D). Interestingly, the majority of cells that were correctly aligned with the microvessel axis, expressed this axis long before touching the wall (Fig. 2F).

Cell axis alignment can be blocked by NPA. To gain insight into the cellular mechanisms underlying axis alignment, the regeneration of protoplasts was followed under the same conditions, but adding a low concentration $(1 \mu \mathrm{M})$ of the phytotropin 1-naphthyl phthalamic 
acid (NPA) to the microfluidic stream. This concentration was based on a previous study, where already $3 \mu \mathrm{M}$ of NPA were sufficient to block division synchrony between the individual cells of a file ${ }^{11}$. We observed that the alignment of the regenerating cell axis with the chamber geometry initiating at day 3 under control conditions (Fig. 2D), was eliminated in presence of NPA at day 4 (Fig. 2E), when the majority of cells had already touched the wall. Only at day 7, a slight preponderance of longitudinal (45\%) over transverse (40\%) alignment became detectable. This is produced by cells that had tilted their orientation after being squeezed against the side walls. Thus, the alignment of cell axis is blocked by low concentrations of NPA, a compound that specifically inhibits auxin-efflux carriers.

Geometrical alignment can be overrun by a flux of NAA, but not by $2,4-D$. In the next step we imposed a gradient of the artificial stable auxins 1-naphthalene acetic acid (NAA) and 2,4dichlorophenoxyacetic acid (2,4-D) that differ with respect to their transport properties ${ }^{12,13}$ : NAA is efficiently transported through the efflux carrier blocked by NPA, 2,4-D is transported only poorly. To exclude potential influence of shear forces, the gradient was administered through mere diffusion by adding $2 \mu \mathrm{l}$ of auxin to one of the inlets and stopping the flow after it has been taken up into the channel. Due to the fact that, so far, no method is available that would allow to measure the local concentration of auxin, we had to rely on mathematical calculation. Thus, the spread of the gradient and its subsequent dissipation was calculated based on the parameters of the microfluidic device (Fig. 3B). Since the diffusion coefficients for NAA and 2,4-D are very similar, the result was almost identical. The gradient of auxin along the channel was predicted to be maximal at half a day after loading and to decay subsequently. Directly after loading, the front of auxin was estimated to be around $5 \mathrm{~mm}$ upstream of the first row of microvessels, i.e. $7 \mathrm{~mm}$ downstream of the joint, such that transverse gradients of auxin were negligible. When the response of the cells to a gradient of NAA was screened, a marked difference along the channel was observed (Fig. 3A): Close to the inlet, at the anterior end of the channel, the majority of cells aligned transversely, i.e. parallel with the short axis of the channel. In contrast, at the posterior channel end, most cells aligned longitudinally, i.e. parallel with the long axis of the channel. Due to this difference in orientation, the cells near the inlet were rounder, whereas the cells near the outlet appeared more elongate. In the central regions of the channel, cells developed discrepant alignments, where an originally transverse axis was overlaid by subsequent alignment in longitudinal orientation. Thus, the cells oriented parallel to the NAA gradient in the upstream regions of the microfluidic channel, whereas in the downstream regions the geometry of the microvessel dominated. To quantify this response, the channel was subdivided into four areas from A, most upstream, to $\mathrm{D}$, most downstream, and the frequencies of transverse versus longitudinal alignment were scored over time (Figs. 4A, B). There was a clear gradient in the frequency of transverse alignment with higher incidences in area A and a steady decrease over areas B and C to minimal values in the downstream area $\mathrm{D}$. This gradient was detectable from day 3 on and became more prominent reaching $55 \%$ in area $\mathrm{A}$ and over $38 \%$ in area D, if scored at day 7. Conversely, the frequency of longitudinal alignments increased from the upstream area A (42\%) to the downstream area D (52\%). The frequency of non-regenerating protoplasts arrested in stage 0 was lower than $10 \%$ throughout. The comparison with the control values in the absence of additional auxin (Fig. 4E, Supplementary Information), shows clearly that the geometrical alignment was strongly reduced in presence of an auxin gradient even in the most remote area $\mathrm{D}$. In the most upstream area $\mathrm{A}$, geometrical alignment was even overrun by the effect of the auxin gradient. For comparison we repeated the same set-up replacing NAA by 2,4-D (Figs. 4C, D). Again, there was a gradient of geometrical alignment with a progressive dominance of longitudinal over transverse orientation from areas A over B, C till area D. However, the reduction of this geometrical alignment by $2,4-\mathrm{D}$ was less pronounced and was not able to overrun the geometrical alignment, in contrast to NAA. Thus, although both artificial auxins could weaken the impact of microvessel geometry, the effect of a gradient of NAA was stronger and even able to override the impact of geometry, whereas the effect of 2,4-D was more limited to a reduction of geometrical alignment.

\section{Discussion}

A technical "tissue" allows to separate mechanical from chemical cues. Plant cells process chemical and mechanical cues for alignment of their axis of growth and division to minimize mechanical tensions in the turgescent tissue. As illustrated by the phyllotaxis model, the individual roles of these cues are difficult to be separated in the complex context of organs and even tissues. This was the motivation for our strategy to mimic both cues by a technical simulation of a plant tissue. Here, the chemical cue, auxin flux, was administered as microfluidic gradient, whereas the directional mechanical cue was achieved by rectangular microvessels of a size that corresponds to the dimensions set by cell walls in a natural tissue. Plant protoplasts as symmetrical cells that had been stripped from their innate directionality by digestion of the cell wall were integrated into this technical framework. Although the idea of a biomimetic array of "artificial cells" that are arranged in a flow of a chemical signal may seem straightforward, the technical realization of this approach was far from trivial. A long preparatory phase was required to reconcile the technical with the biological requirements and to establish a system that could be handled. The system was preserving biological functionality and at the same time allowed for a high degree of reproducibility. Biomimetic microfluidic devices inspired by plant tissues have been generated previously by parallel channel networks embedded in a polymeric material layer and used to investigate the optimal pattern for maximal flow in order to understand the biophysical constraints shaping venation patterns in real leaves ${ }^{14}$, or by generating a microfluidic system in a hydrogel to study biophysical aspects of transpiration ${ }^{15}$. However, these approaches remained merely technical and did not integrate cells. There are two reports, where plant cells- protoplasts from tobacco leaves- have been kept alive in a microfluidic channel over several days ${ }^{16,17}$ - however, in both cases, cells were floating freely in a large compartment of around $1 \mathrm{~mm}$ width without any possibility to administer directional cues to the regenerating protoplasts. In contrast, the system described in the current work successfully integrates living, developing plant cells into a technical realization of a tissue, where either mechanical or chemical cues can convey directional information. To our knowledge, this represents the first example, where this has been achieved for plant cells.

In our previous work, we have followed the role of the cytoskeleton for the symmetry break in the regenerating protoplast ${ }^{9}$. Interestingly, the first phase of regeneration is characterized by extensive nuclear movements. Only when the nucleus has been positioned to the cell center during a long preparatory phase that usually lasts an entire day, the symmetrical cell produces a new cell wall, which is a rapid process completed in a few minutes. This walled cell is still perfectly symmetric and only following that stage symmetry break, observed by alignment of actin in a gradient followed by microtubular alignment perpendicular to this gradient. This scenario supports a model, where mechanical constraints of the cell wall are used as spatial input for cytoskeletal reorganization similar to phyllotaxis in the developing apical meristem ${ }^{5}$. Our observation that the cells align with the longer chamber axis, are congruent with this model. However, a closer look revealed that the alignment with the longer chamber axis became evident, before the cells were touching the wall, i.e. before there was any mechanic input from pressure of the expanding cell against a mechanic constraint. This indicates that the cells use a 


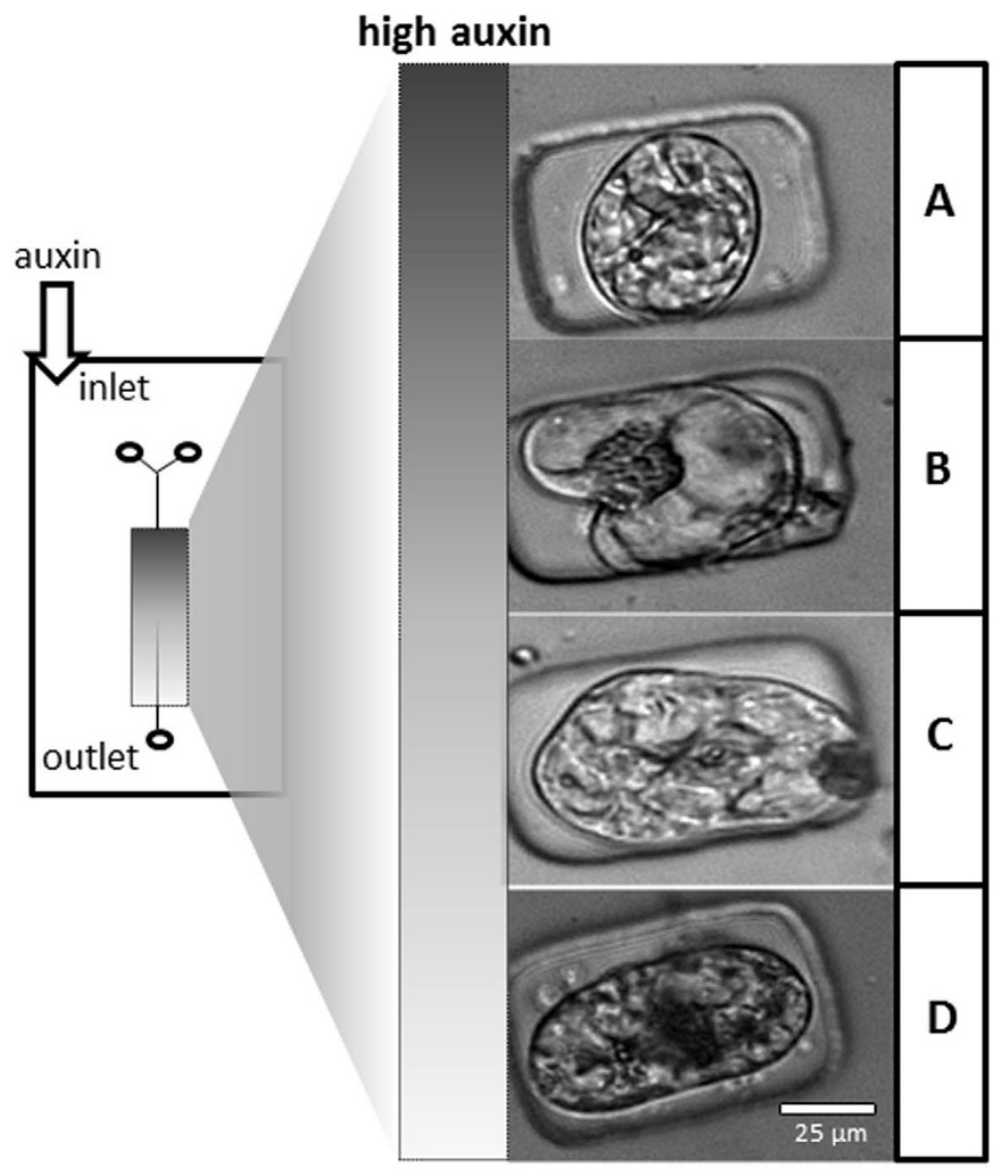

low auxin

B
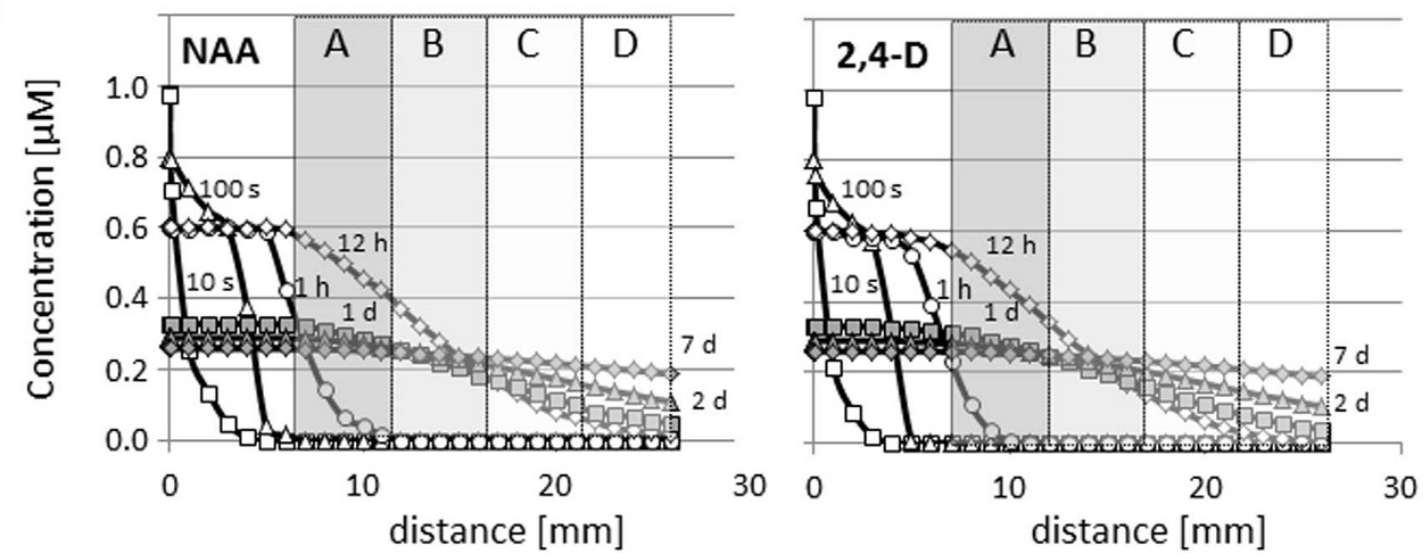

Figure 3 Longitudinal gradients of auxins interfere with geometrical alignment. (A) Set-up of the experiment: the artificial stable auxins 1-naphthalene acetic acid (NAA), or 2,4-dichlorophenoxyacetic acid (2,4-D), where administered to one of the inlets $(8 \mu \mathrm{l}, 1 \mu \mathrm{M})$ after filling the channel with protoplasts. Flux through the channel was stopped, such that the applied auxin dissipated into the channel by diffusion. The resulting gradient was calculated to become maximal around 0.5 days after application and to weaken subsequently slowly approaching a final concentration of around $0.2 \mu \mathrm{M}$ auxin. Cellular responses were observed and scored (Figure 4) in four domains (A-D) of the gradient as indicated in the figure. Representative images for the cellular responses in these domains are shown for treatment with NAA. (B) Predicted temporal dynamics of the auxin gradient.

non-mechanical signal to explore the geometry of the surrounding space. This signal might be auxin. We tested this idea by the inhibition of auxin efflux by the well established and specific inhibitor $\mathrm{NPA}^{18}$. In fact, we observed that the alignment of cell axis with the longer chamber axis was lost in presence of NPA. We arrive at a model, where the expanding protoplasts use auxin efflux into their environment to explore the geometry of their environment and subsequently use differences in the local concentration of auxin to align their axis such that during later expansion the resulting mechanical tension will be minimized. Whether the explorative signal is auxin itself, or whether, alternatively, a signal that interferes with auxin passage through the membrane (an interesting candidate would be apoplastic protons) will be a topic for future experiments.

The discovery of auxin-dependent alignment leads to question on the nature of the input. Principally, the cells might respond to the local concentration of auxin at the different flanks of the regenerating 

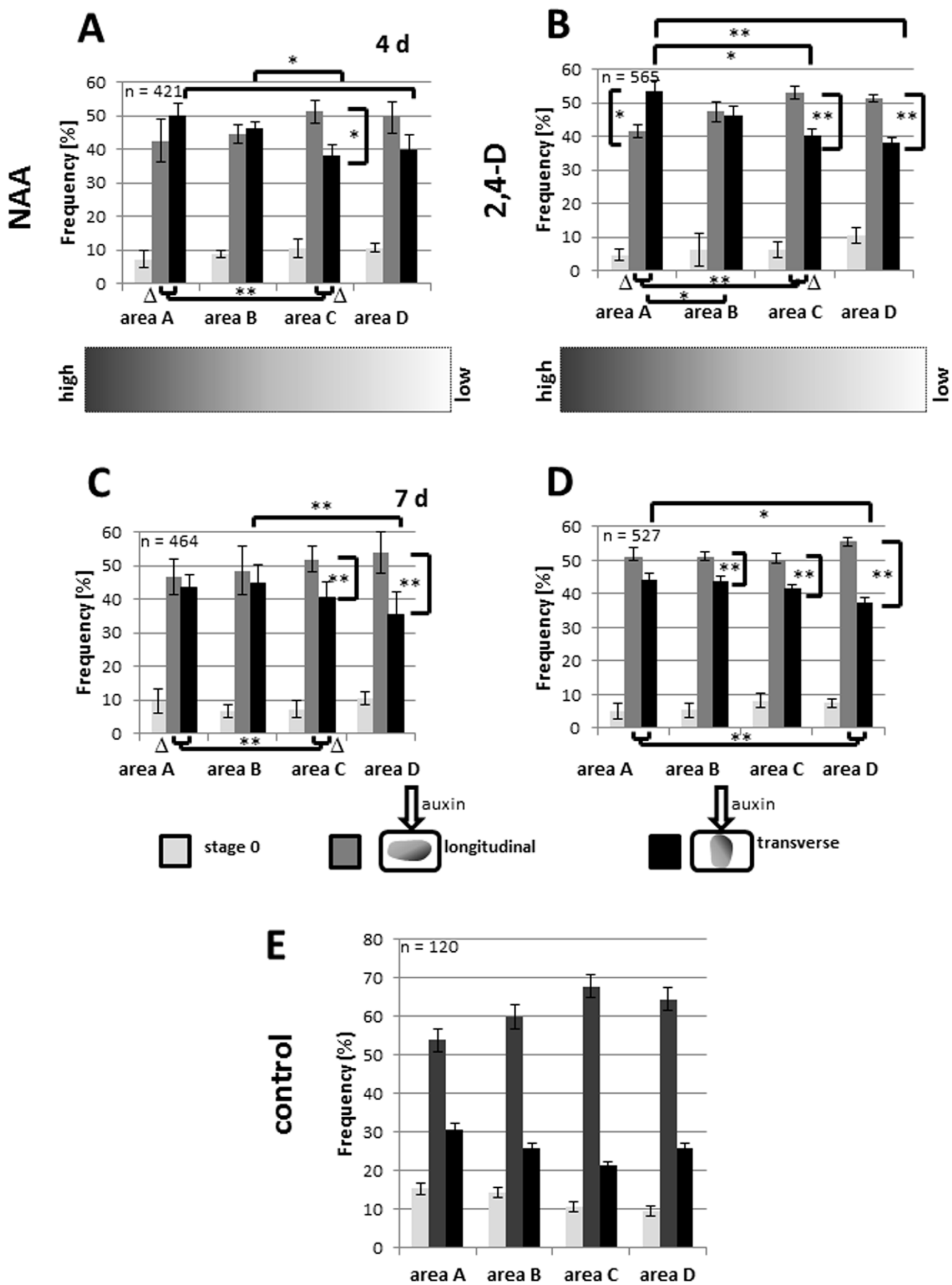

Figure $4 \mid$ Quantification of the interference by gradients of auxins with geometrical alignment. The incidence of longitudinal (grey bars) and transverse (black bars) cells, as well as protoplasts that have not initiated regeneration (stage 0, light grey bars) in the different domains (A-D, see Figure 3) of a gradient imposed by $1 \mu \mathrm{M}$ of the artificial stable auxins 1-naphthalene acetic acid (NAA) (A, B), or 2,4-dichlorophenoxyacetic acid (2,4-D, C, D) at days 4 $(\mathrm{A}, \mathrm{C})$ and 7 (B, D) as compared to controls in the absence of auxins (E) assessed shown for the final time point (day 7). Data represent mean values and standard errors of five independent biological replicas. Brackets represent statistical significance of the indicated differences as tested by a paired, twosided t-test $\left({ }^{*} P<5 \%,{ }^{* *} P<1 \%\right)$, the brackets labelled by $\Delta$ represent statistical significance for the difference between transverse and longitudinal alignment in a given domain. 
cell. Alternatively, they might respond to the flux of auxin that has passed through them. The debate on these two models has been quite controversial since Tsvi Sachs ${ }^{19}$ proposed that the auxin transported through a given cell in the regenerating tissue will act as signal promoting the capacity for polar auxin transport in a positive feedback loop. This so-called auxin canalisation is seen as the core mechanism for the flexible architectural self organisation of vascular land plants: this mechanism will channel auxin flux in regenerating tissues into distinct pathways that later will differentiate into vascular bundles such that new organs as young leaf primordia or buds will be optimally connected, but also interruptions by wounds inflicted onto the pre-existing vasculature can be bypassed. Microscopical details of fossil wood suggest that this canalisation has been used back to 400 mya ago $^{20}$. A conceptual problem of this flux canalisation model has been the need to explain, how a cell could measure the flux of auxin (a "holistic feature"). Therefore, alternative mechanisms have been searched that would sense local concentrations of auxin as input rather than fluxes of auxin through the cell ${ }^{21}$ : The polarity of auxin flux is brought about by a transcellular gradient in the activity of auxin efflux carriers such as the PIN transporters. This gradient of PIN activity has been shown to arise from a gradient in PIN cycling between the plasma membrane and intracellular stores ${ }^{22}$. This cycling, in turn, depends on the concentration of auxin in the environment $^{23}$, and on dynamic actin filaments that are remodelled in response to auxin (for review see 24). When the cycling of PINs would differ as a function of local auxin concentration, this would explain in principle, why the cell axis aligns with auxin gradients and would circumvent the need to sense fluxes of auxin. Since the gradient is maximal at $0.5 \mathrm{~d}$ after loading and dissipates later, the induction of a cell axis in parallel to auxin is determined during the first day of regeneration, which is consistent with experiments, where polarity in regenerating BY-2 protoplasts was disturbed by inducible expression of actin-bundling proteins at different time points ${ }^{9}$. Interestingly, this axis becomes manifest only from $2 \mathrm{~d}$ later.

We wondered, whether our "artificial tissue" might contribute to this debate and therefore administered a gradient of two artificial auxins that differ with respect to their transport features: NAA is transported in a polar manner very similar to the natural auxin IAA, whereas $2,4-\mathrm{D}$ is transported only very poorly ${ }^{12,13}$. The auxin gradient was oriented perpendicular with the long axis of the microvessels, and we asked, whether this gradient could affect or possibly overrun the directional cues derived from microvessel geometry. For NAA, this was exactly what we observed. In contrast, a gradient of 2,4-D failed to overrun the effect of microvessel geometry. This indicates that directional transport of a given auxin species promotes the alignment of cell axis with the auxin gradient. Interestingly, NAA, but not 2,4-D, was found to be effective in triggering the actin remodelling required to sustain polar auxin transport as shown for tobacco BY $-2^{11}$ and rice coleoptiles ${ }^{26}$. There is a second argument in favour of the auxin-flux mechanism: The local sensing mechanism predicts that PIN transporters would accumulate at the sites of elevated auxin concentration such that auxin is pumped up into local maxima ${ }^{21}$. When the expanding protoplast approaches the wall of the microchamber, this should cause a local increase of the secreted auxin in the limited space between membrane and chamber wall, such that PIN transporters would be expected to accumulate there. This should result in axis alignment parallel to the shorter axis of the microchamber - opposite to our observations. As experimental approach to discriminate between different sensing mechanisms, the auxinreporter line DR5::GFP could be integrated into the gradient. However, it should be kept in mind that gradients potentially visualized by this reporter would not necessarily monitor local concentrations of auxin, but the temporal integral of auxin-dependent transcriptional activation. A further strategy would be to use 2NAA as well transportable, but biologically inactive analogue, as well as other inhibitors such as CPD (1-(2-carboxyphenyl)-3-phenyl-pro- pane-1,3-dione), PBA (1-pyrenoylbenzoic acid), Brefeldin A, or TIBA (2,3,5-triiodobenzoic acid).

Conclusions and outlook. Using a microfluidic based technical simulation of a tissue and regenerating protoplasts we investigated, how plant cells adjust their axis in a way that resulting mechanical tensions in the tissue will be minimized. We show that the regenerating protoplast form a new axis in harmony with the geometry of their environment. To explore geometry, they use auxin as chemical cue, before facing constraints from physical contact with the wall of the vessel (or, in real tissues, the counterforce from a cell wall). This chemical sensing of geometry requires auxin efflux with input from local auxin concentration. A gradient of 2,4-D, although less efficient than NAA, can reduce to a certain extent the input from microvessel geometry.

This system is of course still a very rough approximation of the situation in a tissue, where local maxima and minima convey positional information. To further dissect supracellular versus intracellular gradients, feeding of caged IAA and subsequent local release of active auxin by means of micro irradiation would be promising ${ }^{27}$. This can then be combined with transgenic cell lines, where either cytoskeletal components ${ }^{9}$ or components of auxin transport and signalling are visualized by fluorescent proteins.

\section{Methods}

Fabrication of the microfluidic device. The microchannels and microvessels were fabricated in polydimethylsiloxane (PDMS) using soft lithography and replica molding. A layer of photoresistant SU-8 2100 (MicroChem Corp., MA, US) was patterned on a silicon wafer by photolithography, resulting in a positive relief patterns of channels and a series of relief patterns of microvessels. For channels, a prepolymer mixture of Sylgard 184 (Dow Corning, MI, US) was cast and cured against the positive relief master to obtain a negative replica-molded piece. After curing for $2 \mathrm{~h}$ at $70^{\circ} \mathrm{C}$, the PDMS was peeled off the master, and inlets and outlets of the channel were punched with a sharpened needle. For microvessels, a spin coater (1000 rpm, $10 \mathrm{~s})$ was used to cure a prepolymer mixture of Sylgard 184 (Dow Corning, MI, US) against the positive relief master to obtain a negative replica-molded membrane. After curing for $10 \mathrm{~min}$ at $70^{\circ} \mathrm{C}$, the membrane was peeled off the master, and the upper surface that had no pattern was adhered to glass. The patterned surfaces of channels and microvessels were treated with oxygen plasma for 1 min with $50 \mathrm{~mW}$, and, subsequently, the microchannel was assembled on top of the microvessels to form an integrated device. The dimensions of an individual microvessel were $72.8 \pm 1.36 \mu \mathrm{m}$ in length and $52.5 \pm 0.38$ in width, the filled channel kept $9.5 \mu \mathrm{l}$ in volume in total.

Plant material and protoplasts. The cell strain BY-2 (Nicotiana tabacum L. cv Bright Yellow-2; 28) was cultivated as suspension in liquid medium containing $4.3 \mathrm{~g} / \mathrm{L}$ Murashige and Skoog (MS) salts (Duchefa Biochemie), $30 \mathrm{~g} / \mathrm{L}$ sucrose, $200 \mathrm{mg} / \mathrm{L}$ $\mathrm{KH}_{2} \mathrm{PO}_{4}, 100 / \mathrm{mg} / \mathrm{L}$ (myo)-inositol, $1 \mathrm{mg} / \mathrm{L}$ thiamine, and $0.2 \mathrm{mg} / \mathrm{L} 2,4-\mathrm{D}, \mathrm{pH} 5.8$. The cells were subcultivated weekly, inoculating $1.0 \mathrm{~mL}$ of stationary cells into fresh medium $(30 \mathrm{~mL})$ in 100-mL Erlenmeyer flasks. The cells were incubated in darkness at $27^{\circ} \mathrm{C}$ under constant shaking on a KS260 basic orbital shaker (IKA Labortechnik, Germany) at $150 \mathrm{rpm}$. Protoplasts were generated as described in Zaban et al. (2013) and regenerated in FMS store medium (4.3 g/L MS- salts, $100 \mathrm{mg} / \mathrm{L}$ (myo)-inositol, $0.5 \mathrm{mg} / \mathrm{L}$ nicotinic acid, $0.5 \mathrm{mg} / \mathrm{L}$ pyroxidine- $\mathrm{HCl}, 0.1 \mathrm{mg} / \mathrm{L}$ thiamin and $10 \mathrm{~g} / \mathrm{L}$ sucrose in $0.25 \mathrm{M}$ mannitol complemented with $0.1 \mathrm{mg} / \mathrm{L} 1$-naphthaleneacetic and $1 \mathrm{mg} / \mathrm{L}$ benzylaminopurin). For cellulose staining, Calcofluor White $(0.1 \% \mathrm{w} / \mathrm{v})$ was directly added to the protoplast suspension ${ }^{29}$.

Charging and compartmentation of microchannels. The microchannels were fixed onto a microscopic slide and filled with a micropipette with FMS store medium. Subsequently, the microchannels were degased by generating a vacuum in an exsiccator using a water- jet vacuum pump. $5 \mu \mathrm{l}$ of protoplast suspension were filled into the left inlet of the microchannel via a microfluidic pump (MS-CA 4/820, Ismatec, Wertheim; Germany) by a slow flow $\left(0.021 \mathrm{ml} . \mathrm{h}^{-1}\right)$. To generate auxin gradients, the channel was loaded with cells, and then the flow stopped. Subsequently, $2 \mu \mathrm{l}$ of $1 \mu \mathrm{M}$ NAA or 2,4 D in FMS store medium were administered into the right inlet such that a diffusion gradient developed along the axis of the channel. To score the response, the channel was subdivided equally into 4 areas designated $A$ to $D$, with A located upstream in the gradient. The temporal dynamics of the auxin gradient was calculated based on Fick's second law in one dimension based on a diffusion coefficient for NAA of $6.6 \cdot 10^{-6} \mathrm{~cm}^{2}$. $\mathrm{sec}^{-130}$ and $7.98 \cdot 10^{-6} \mathrm{~cm}^{2} . \mathrm{sec}^{-1}$ for $2,4-\mathrm{D}^{31}$.

Microscopy and quantification of the response. After covering the loaded microvessels with a coverslip, they were examined under an Axioskop microscope (Zeiss, Jena, Germany), equipped with a 32x long distance objective (Zeiss Neofluar, Jena, Germany), and a digital CCD camera (AxioCam MRm). The Calcofluor White signal was recorded through the filter set 49 (excitation at $365 \mathrm{~nm}$, beamsplitter at 
$395 \mathrm{~nm}$, and emission at $445 \mathrm{~nm}$; Zeiss, Jena, Germany). Images were processed and analyzed using the AxioVision software (Rel. 4.8.2) (Zeiss, Jena, Germany). For the viability assays, between $100-250$ cells were observed per data point. Between 181350 cells were scored per data point for testing the geometry sensing of regenerating protoplast, and the loss of geometry sensing by inhibition of auxin efflux (Fig. 2). For the auxin-gradient analysis (Fig. 4) and cell geometry alterations by auxin gradient $62-186$ cells were counted per area and time point, giving a total population of 408 688 individual cells per time point.

1. Niklas, K. J. \& Spatz, H. C. Growth and hydraulic (not mechanical) constraints govern the scaling of tree height and mass. Proc Natl Acad Sci USA 101, 15661-15663 (2004)

2. Lintilhac, P. M. \& Vesecky, T. B. Stress-induced alignment of division plane in plant tissues grown in vitro. Nature 307, 363-364 (1984).

3. Green, P. B. Organogenesis - a biophysical view. Annu Rev Plant Physiol 3, 51-82 (1980).

4. Fleming, A. J., McQueen-Mason, S. \& Mandel, T. Induction of Leaf Primordia by the Cell Wall Protein Expansin. Science 276, 1415-1418 (1997).

5. Hamant, O. et al. Developmental patterning by mechanical signals in Arabidopsis. Science, 22, 1650-1655 (2008).

6. Reinhardt, D. et al. Regulation of phyllotaxis by polar auxin transport. Nature 426, 255-260 (2003).

7. Reinhardt, D., Mandel, T. \& Kuhlemeier, C. Auxin regulates the initiation and radial position of plant lateral organs. Plant Cell 12, 507-518 (2000).

8. Guenot, B., Bayer, E., Kierzkowski, D., Smith, R. S., Mandel, T., Zádníková, P., Benková, E. \& Kuhlemeier, C. PIN1-Independent Leaf Initiation in Arabidopsis. Plant Physiol 159, 1501-1510 (2012).

9. Zaban, B., Maisch, J. \& Nick, P. Dynamic actin controls polarity induction de novo in protoplasts. J Int Plant Biol 55, 142-159 (2013).

10. Sun, Y., Liu, Y., Qu, W. \& Jiang, X. Combining nanosurface chemistry and microfluidics for molecular analysis and cell biology. Anal Chim Acta 650, 98-105 (2009).

11. Maisch, J. \& Nick, P. Actin is involved in auxin-dependent patterning. Plant Physiol 143, 1695-1704 (2007).

12. Delbarre, A., Meller, P., Imhoff, V. \& Guern, J. Comparison of mechanisms controlling uptake and accumulation of 2,4-dichlorophenoxy acetic acid, naphthalene-1-acetic acid, and indole-3-acetic acid in suspension cultured tobacco cells. Planta 198, 532-541 (1996)

13. Hošek, P., Kubeš, M. \& Laňková, M. Auxin transport at cellular level: new insights supported by mathematical modelling. J Exp Bot 63, 3815-3827 (2012).

14. Noblin, X. et al. Optimal vein density in artificial and real leaves. Proc Natl Acad Sci USA 105, 9140-9144 (2008).

15. Wheeler, T. D. \& Stroock, A. D. The transpiration of water at negative pressures in a synthetic tree. Nature 455, 208-212 (2008).

16. Ko, J. M., Ju, J. I., Lee, S. H. \& Cha, H. C. Tobacco protoplast culture in a polydimethylsiloxane-based microfluidic channel. Protoplasma 227, 237-240 (2006).

17. Wu, H. et al. Culture and chemical-induced fusion of tobacco mesophyll protoplasts in a microfluidic device. Microfluid Nanofluid 10, 867-876 (2011).

18. Thompson, K. S., Hertel, R., Müller, S. \& Tavares, J. E. 1-N-naphthylphthalamic and 2,3,5-triiodobenzoic acids: in vitro binding to particulate cell fractions and action on auxin transport in corn coleoptiles. Planta 109, 337-352 (1973).

19. Sachs, T. Polarity and the induction of organized vascular tissues. Ann Bot 33, 263 (1969)
20. Rothwell, G. W. \& Lev-Yadun, S. Evidence of polar auxin flow in 375 million-yearold fossil wood. Am J Bot 92, 903-906 (2005).

21. Merks, R. M. H., Van de Peer, Y., Inzé, D. \& Beemster, G. T. S. Canalization without flux sensors: a traveling-wave hypothesis. Trends Plant Sci 12, 384-290 (2007).

22. Dhonukshe, P. et al. Generation of cell polarity in plants links endocytosis, auxin distribution and cell fate decisions. 0 456, 962-966 (2008).

23. Paciorek, T. et al. Auxin inhibits endocytosis and promotes its own efflux from cells. Nature 435, 1251-1256 (2005).

24. Nick, P. Probing the actin-auxin oscillator. Plant Signaling Behav 5, 4-9 (2010).

25. Goodner, B. \& Quatrano, R. S. Fucus embryogenesis: A model to study the establishment of polarity. Plant Cell 5, 1471-1481 (1993).

26. Nick, P., Han, M. \& An, G. Auxin stimulates its own transport by actin reorganization. Plant Physiol 151, 155-167 (2009).

27. Kusaka, N. et al. Manipulation of Intercellular Auxin in a Single Cell by Light with Esterase-Resistant Caged Auxins. ChemBioChem 10, 2195-2202 (2009).

28. Nagata, T., Nemoto, Y. \& Hasezawa, S. Tobacco BY-2 cell line as the "HeLa" cell in the cell biology of higher plants. Int Rev Cytol 132, 1-30 (1992).

29. Maeda, H. \& Ishida, N. Specificity of binding of hexopyranosyl polysaccharides with fluorescent brightener. J Biochem 62, 276-278 (1967).

30. Duan, C. Q., Zhang, Y. M. \& Gao, Z. N. Electrochemical Behaviors and Electrochemical Determination of 1-naphthaleneacetic Acid at an Ionic Liquid Modified Carbon Paste Electrode. Proc Natl Acad Sci USA 78, 976-980 (2012).

31. Saxena, S. K., Boersma, L., Lindstrom, F. T. \& Young, J. L. The Self-Diffusion Coefficients of ${ }^{45} \mathrm{Ca}$ and 2,4-Dichlorophenoxyacetic Acid. Soil Science 117, 14-20 (1974).

\section{Acknowledgments}

This work has been supported by a collaborating grant from the SinoGerman Science Center (project GZ614 "Probing self-organization by microfluidics"). Methodological advice from Dr. J. Maisch (KIT) is gratefully acknowledged.

\section{Author contributions}

P.N. wrote the main manuscript text and prepared the figures, B.Z. did the research based on the protoplasts with data collection and photos, W.L. and X.J. fabricated the microfluidic devices. All authors reviewed the manuscript.

\section{Additional information}

Supplementary information accompanies this paper at http://www.nature.com/ scientificreports

Competing financial interests: The authors declare no competing financial interests.

How to cite this article: Zaban, B., Liu, W., Jiang, X. \& Nick, P. Plant Cells Use Auxin Efflux to Explore Geometry. Sci. Rep. 4, 5852; DOI:10.1038/srep05852 (2014).

This work is licensed under a Creative Commons Attribution-NonCommercialNoDerivs 4.0 International License. The images or other third party material in this article are included in the article's Creative Commons license, unless indicated otherwise in the credit line; if the material is not included under the Creative Commons license, users will need to obtain permission from the license holder in order to reproduce the material. To view a copy of this license, visit http:// creativecommons.org/licenses/by-nc-nd/4.0/ 